\title{
Fibrinogen-Based Collagen Fleece Graft Myringoplasty for Traumatic Tympanic Membrane Perforation
}

\author{
Seung Hyo Choi ${ }^{1}$, Hyoung Yong Song ${ }^{2}$, and Chan II Song ${ }^{1}$ \\ ${ }^{1}$ Department of Otorhinolaryngology-Head and Neck Surgery, Jeju National University School of Medicine, Jeju, \\ 2Department of Otorhinolaryngology, Gwangmyeong Sungae Hospital, Gwangmyeong, Korea
}

$\begin{array}{ll}\text { Received } & \text { March 15, 2016 } \\ \text { Revised } & \text { May 19, 2016 } \\ \text { Accepted } & \text { June 7, 2016 }\end{array}$

Address for correspondence

Chan Il Song, MD

Department of Otorhinolaryngology-

Head and Neck Surgery,

Jeju National University

School of Medicine,

15 Aran 13-gil, Jeju 63241, Korea

Tel $+82-64-717-2108$

Fax +82-64-717-1029

E-mailssongchanil@gmail.com
Background and Objectives: The aim of this study was to investigate how fibrinogenbased collagen fleece $\left(\right.$ Tachocomb ${ }^{\circledR}$ ) graft myringoplasty (FCGM), performed under microscopic guidance, improves both hearing and tympanic membrane tissue repair in patients with traumatic tympanic membrane perforation (TMP). Subjects and Methods: Between August 2009 and March 2015, a total of 52 patients with traumatic TMP visited the department of otorhinolaryngology at a secondary medical center. Twenty-nine of these underwent FCGM under microscopic guidance in our outpatient clinic. For each patient, we recorded the location and size of the perforation, the time elapsed from the onset of TMP until the myringoplasty, and the hearing level both before and after myringoplasty. Results: The TMP closed completely in all cases (29 of 29 patients). After myringoplasty, the postoperative airbone gap (ABG) differed significantly from the preoperative ABG. Three of the 29 patients (10.3\%) experienced complications. Specifically, 2 presented with otorrhea after FCGM, but conservative management led to improvement without recurrence of perforation. One patient showed delayed facial palsy 1 week after the procedure. The condition of this patient also improved and the palsy was not permanent. Conclusions: FCGM may be an effective treatment option in case of traumatic TMP. The procedure requires no hospitalization, and can be used to avoid traditional tympanoplasty.

J Audiol Otol 2016;20(3):139-145

KEY WORDS: Tympanic membrane perforation · Fibrinogen-based collagen fleece . Myringoplasty $\cdot$ Conductive hearing loss $\cdot$ Patch.

\section{Introduction}

The tympanic membrane perforation (TMP) can be classified according to their duration, in acute and chronic (more than 3 months), and by the presence or absence of drainage, in wet and dry perforation [1]. In recent times, the number of patients presenting with chronic otitis media has been decreasing rapidly due to advances in antibiotic treatment. For this reason, trauma has become one of the main causes of TMP. While most patients of traumatic TMP recover spontaneously without complications [2], some suffer middle ear infections and develop conductive hearing problems caused by unhealed perforations [3]. Although physicians often at-

This is an Open Access article distributed under the terms of the Creative Commons Attribution Non-Commercial License (http://creativecommons.org/licenses/by-nc/3.0/) which permits unrestricted non-commercial use, distribution, and reproduction in any medium, provided the original work is properly cited. tempt a suitable non-surgical treatment of TMP at an early stage, invasive surgery such as tympanoplasty or myringoplasty is nonetheless required in cases with insufficient healing. Such surgery is more expensive, involves more effort, and entails higher risks than non-surgical treatments. Therefore, treatment using substances that facilitate membrane regeneration has recently been considered as an alternative to surgical TMP repair [4].

The fibrinogen-based collagen fleece $\left(\right.$ Tachocomb $^{\circledR}, \mathrm{Ny}-$ comed Austria GmbH, Linz, Austria) is a collagen-bound fibrinogen sealants. It consists of a sponge-like patch composed of equine collagen and coated with a mixture of human fibrinogen, bovine thrombin, and bovine aprotinin [5]. It has been used both to stop bleeding and to treat leakage of cerebrospinal fluid, bile, saliva, pleural effusion, and lymph. It is also well known that the fibrinogen-based collagen fleece adheres strongly to tissue and thereby forms a waterproof mem- 
brane. Collagen types I to IV are the most abundant collagens in animals. For example, muscular fascia, which is commonly used to repair perforated tympanic membrane (TM) tissue during myringoplasty, consists primarily of collagen type I [6]. In addition, collagen types I and III have been found at the perforation border and around dilated blood vessels early in the healing phase after myringotomy in rats [7]. We decided to use the fibrinogen-based collagen fleece as a patch material because it has 1) good tissue affinity, 2) water-proofing properties, and 3) abundant type I collagen.

The aim of this study was to present the clinical outcomes of fibrinogen-based collagen fleece graft myringoplasty (FCGM) in traumatic TMP patients. The procedure was performed under microscopic guidance in our outpatient clinic.

\section{Subjects and Methods}

\section{Subjects}

Between August 2009 and March 2015, 52 patients with traumatic TMP visited the department of otorhinolaryngology at a secondary medical center. Of these, 29 underwent FCGM under microscopic guidance at the outpatient clinic. Ten patients were loss to follow-up after diagnosis of TMP, and 3 patients discontinued to visit the hospital after FCGM. Six patients were excluded because of the small TMP (less than $30 \%$ of the entire TM area), and 4 patients were young age (3-8 years old). For each patient, we recorded the location and size of perforation, the time elapsed from the onset of TMP until the myringoplasty, and the hearing level both before and after myringoplasty. There were 17 male and 12 female patients ranging in age from 13 to 69 years (mean age, $33.3 \pm 16.6$ years). Indications for FCGM under microscopic guidance were as follows: patients 1) had a direct injury to the TM wherein the perforation area measured more than $30 \%$ of the entire TM area, and 2) the size of TMP was more than half of its original size after 2 weeks of observation. We also included patients in whom TMP had recurred despite their having undergone the paper patch procedure. All patients included in this study consented to participate in the study after hearing the merits and demerits of FCGM. We evaluated the patients using pure tone audiometry (PTA) both before the procedure and after the TMP had completely healed.

\section{Surgical technique}

The surgical procedure, FCGM under microscopic guidance, was carried out as follows.

1) The external auditory canal (EAC) was cleaned and, along with the TM, locally anesthetized using a $10 \%$ lidocaine spray (Xylocaine ${ }^{\circledR} 10 \mathrm{mg}$ spray, Astra Zeneca, Södertälje,

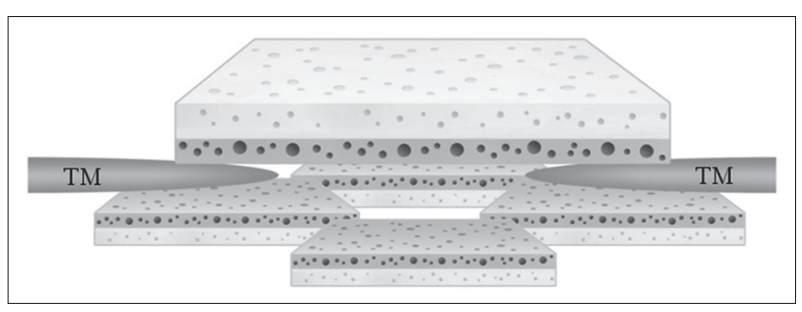

Fig. 1. Schematic illustration of how to place the pieces of the fibrinogen-based collagen fleece. The smaller pieces of the fibrinogen-based collagen fleece were inserted into the middle ear cavity, and the larger piece was placed onto the lateral side of TM. TM: tympanic membrane.

Sweden). Ten minutes were allowed to pass between application of the spray and continuation of the surgery.

2) We divided the fibrinogen-based collagen fleece into pieces of about the size of the perforation, and one piece of about twice the size.

3) The TM was inspected, and the edge of the perforation was unfolded using the ear hook if necessary.

4) We inserted the smaller pieces of the fibrinogen-based collagen fleece into the middle ear cavity, and placed the larger piece onto the lateral side of TM (Fig. 1). Fibrinogen-based collagen fleece patch was not removed until it was detached from the TM by itself.

\section{Audiological test}

PTA was performed before and after FCGM, and the frequencies 250, 500, 1,000, 2,000, and 4,000 Hz were used for analysis. The mean air-bone gap (ABG) was defined as the arithmetical average derived from the analysis of these frequencies. After the patch was removed and the TMP was examined the PTA measurements of each patient were recorded.

\section{Statistical analyses}

The data were analyzed using PASW ${ }^{\circledR}$ statistics 18 software (SPSS Inc., Chicago, IL, USA). The Wilcoxon signed rank test was used to compare the preoperative with the postoperative mean ABG, calculated from the PTA recordings. $p$-values $<0.05$ were considered statistically significant.

\section{Institutional review board approval}

This retrospective study was approved by the Institutional Review Board of Jeju National University Hospital for data collection and analysis (IRB No. JEJUNUH 2014-05-022). The requirement for informed consent was waived.

\section{Results}

The location of the TM perforation was classified as anterior, central, posterior, or in multiple positions. Thirteen (44.8\%) 


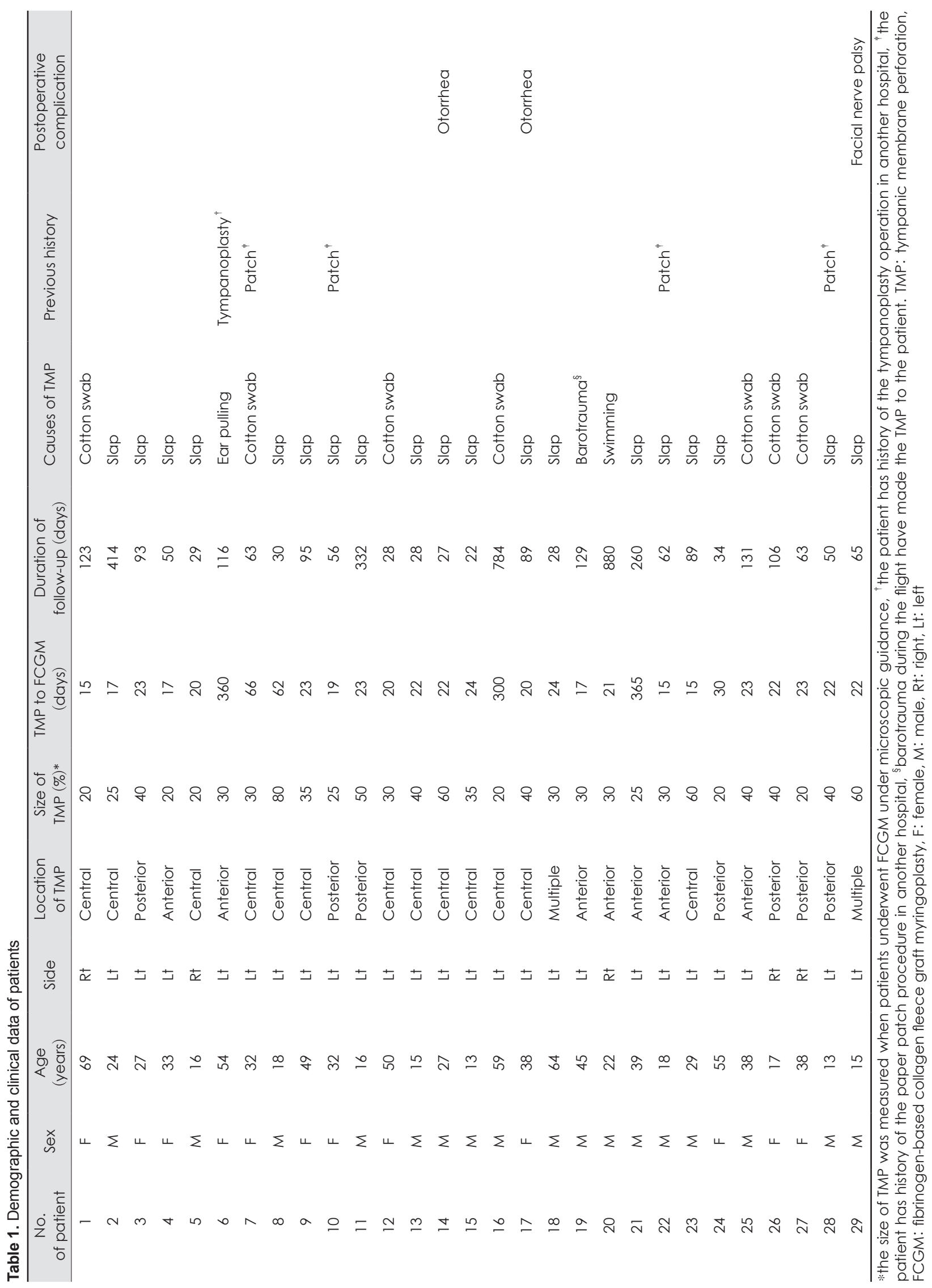


perforations occurred in the central position; 7 (24.1\%) in the posterior position; $7(24.1 \%)$ in the anterior position; and 2 $(6.9 \%)$ in multiple positions. The average perforation area, expressed as a proportion of the entire TM, was $35.3 \pm 14.8 \%$. The elapsed time between the onset of the TM perforation and the hospital visit ranged from 15 days to 12 months $(57.0 \pm$ 99.6 days).

In 19 patients $(65.5 \%)$, the TM was perforated after others had slapped the face. Cotton swabs perforated the TM in a further 7 patients $(24.1 \%)$, and 4 patients (13.8\%) visited our hospital after failure of the paper patch procedure at another hospital.

The overall technical success rate was $100 \%$. That is, all 29 patients showed complete healing of the TM. The complication rate was $10.3 \%$ (3 of 29 patients). Specifically, otorrhea occurred in 2 patients, but they improved without recurrence of perforation. One patient developed delayed facial palsy 1 week after the procedure, but the patient improved and no permanent facial palsy occurred. The mean follow-up period was $147.5 \pm 210.9$ days (28-784 days, Table 1).

When the postperative audiological outcomes in all patients were compared with the preoperative, there was signifi- cant difference with regard to the mean ABGs of patients ( $p<$ $0.001)$. When calculated using frequencies of $500-4,000 \mathrm{~Hz}$, the mean preoperative $\mathrm{ABG}$ was $12.9 \mathrm{~dB} \mathrm{HL}$, whereas the mean postoperative ABG was $2.4 \mathrm{~dB}$ HL. Moreover, ABGs differed significantly at each frequency from $250 \mathrm{~Hz}$ to 4,000 $\mathrm{Hz}(p<0.001)$ (Fig. 2). Fig. 3 shows a representative example of both PTA results and TM findings taken from one of 29 patients.

\section{Discussion}

Both the pars flaccida and the pars tensa of the TM consist of an epidermal layer, a lamina propria, and a mucosal epithelial layer. The fibrils of the fibrous layer contain a large amount of type II and type III collagens, and a small amount of type I collagen. It has been suggested that such an unusual collagen composition is the underlying reason for the unique physical features of the pars tensa of the TM [8]. TMP heals often into a thin atrophic scar which lacks the lamina propria, having only an outer epidermal layer and inner mucosal epithelial layer. This may rupture easily by external trauma, forcible inflation of the Eustachian tube and in otitis media

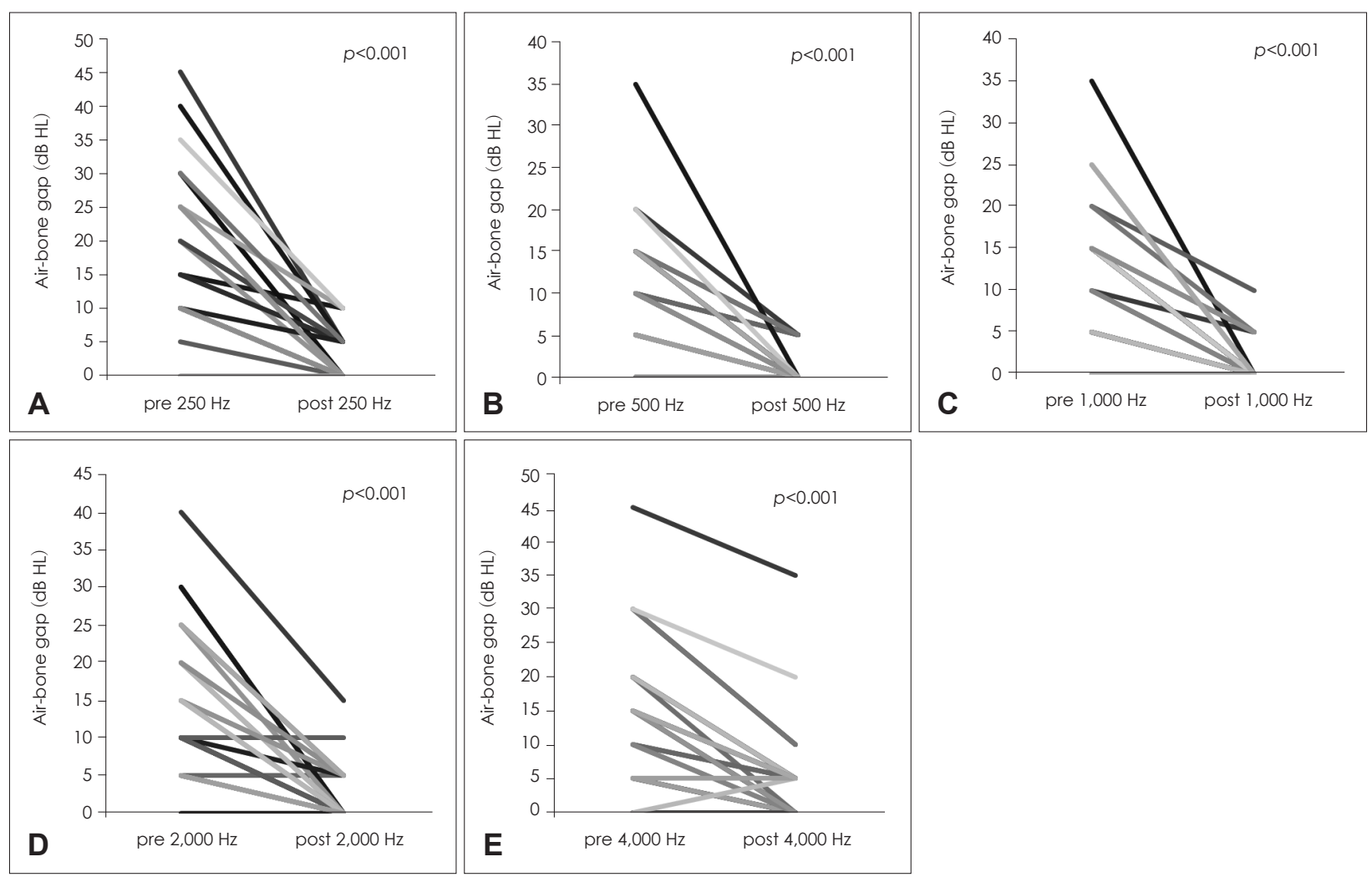

Fig. 2. The change of air-bone gap (ABG) before and after the fibrinogen-based collagen graft myringoplasty (FCGM) at each frequency. ABGs differed significantly at each frequency from $250 \mathrm{~Hz}$ to $4,000 \mathrm{~Hz}$. Twenty-nine patients are represented by each line. A: The change of $A B G$ before and after the FCGM at $250 \mathrm{~Hz}$. B: The change of ABG before and after the FCGM at $500 \mathrm{~Hz}$. C: The change of $A B G$ before and after the FCGM at 1,000 Hz. D: The change of ABG before and after the FCGM at 2,000 Hz. E: The change of ABG before and after the FCGM at $4,000 \mathrm{~Hz}$. ABG: air-bone gap, pre: preoperative, post: postoperative. 
Fig. 3. The PTA results and TM findings of a patient before $(A)$ and after (B) the FCGM. The arrowheads indicate the perforation of TM. PTA: pure tone audiometry, TM: tympanic membra-ne, FCGM: fibrinogen-based collagen fleece graft myringoplasty.
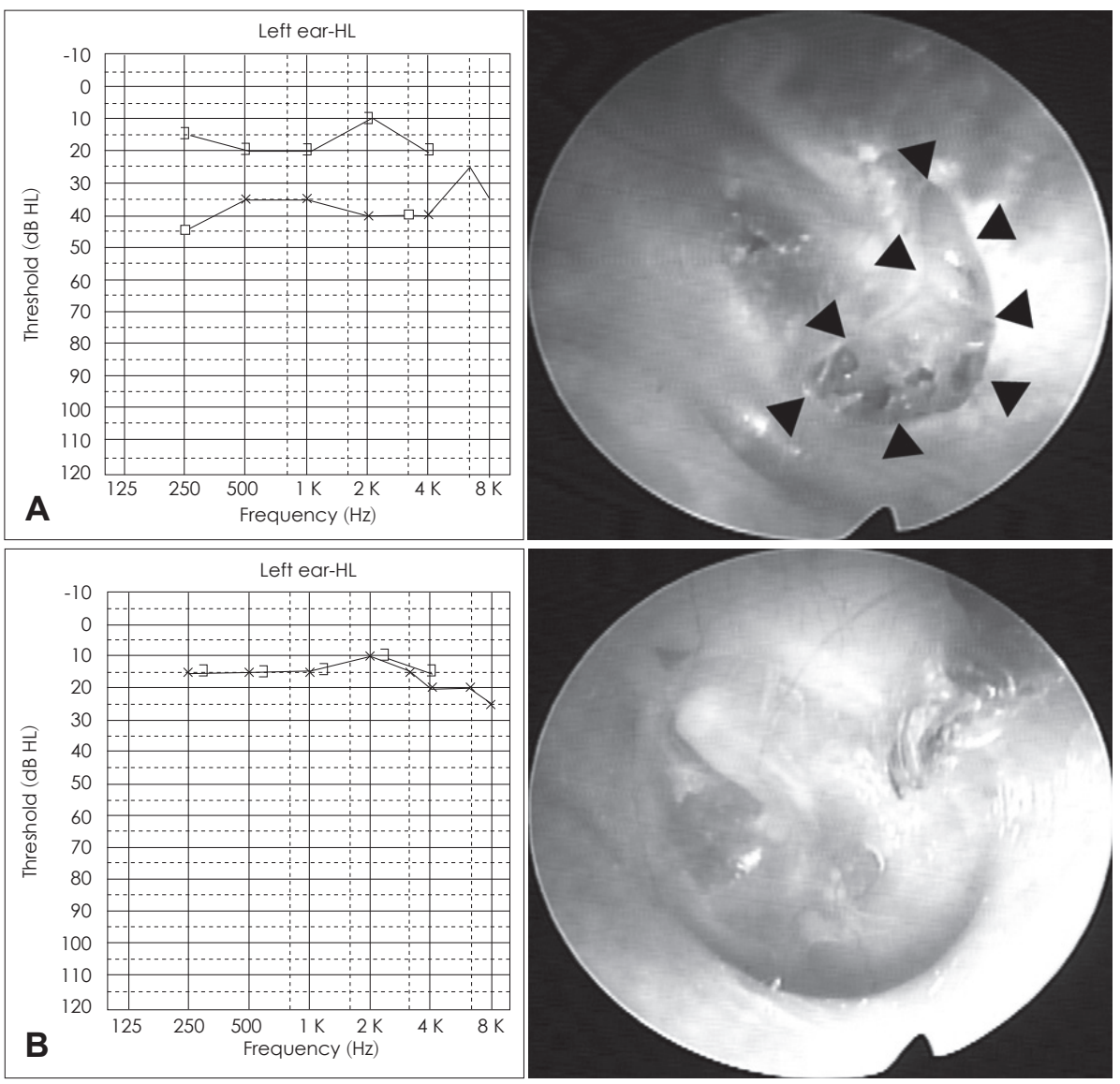

[9]. Histopathological study of a newly formed TMP shows proliferation of squamous epithelium within 12 hours at the edge of the perforation, granulation formation within 18 hours, while the inner mucosa of the membrane takes several days to regenerate [10]. This is one of contributing factors for a perforation to persist. The hypothesis of FCGM is that when on application, the fibrinogen-based collagen fleece may provide collagen for new tissue formation of the lamina propria and act as a splint to bridge the margins of the perforation.

Collagen type I and III are present in the acute healing phase after myringotomy and infection, and the collagen content of the TM is modified during the inflammatory and healing processes [7]. TMP is generally located in the pars tensa. Type II collagen is a main constituent of the lamina propria of the pars tensa, whereas type I collagen is mainly found in the pars flaccida. It follows that graft material in which type II collagen is abundant may more effectively treat TMP in the pars tensa than does our fibrinogen-based collagen fleece, which contains type I collagen. Unfortunately however, we were unable to find commercially-available product containing type II collagen that could be used as a graft material in myringoplasty. Instead of the material with type II collagen, the fibrinogen-based collagen fleece contain- ing type I collagen were used for myringoplasty. The fibrinogen-based collagen fleece is at hand, manageable, and easily stored. It is well known that the commercially-available fibrinogen-based collagen fleece have very few side effects, because it have been widely used. In addition, for FCGM, clinicians need little preparation because of the commerciallyavailable products. Furthermore, in a study involving a model of pleural defect, the fleece seemed to have had completely dissolved after 12 weeks [11].

There was no control group in this study. However, the results of FCGM are better than those of conventional myringoplasty or paper patching in the literatures (overall success rate of $88.9-90.9 \%$ ) [3,12]. The audiological outcomes of paper patching procedure showed the mean preoperative $A B G$ of $23.6 \mathrm{~dB} \mathrm{HL}$, whereas the mean postoperative $\mathrm{ABG}$ of 1 $\mathrm{dB}$ HL [3]. In addition, the overall success rates of "no therapy" are lower than $80 \%$ in previous studies [3,12-14]. Those patients with no therapy showed a preoperative $A B G$ was $26.1 \mathrm{~dB}$ HL, whereas the mean postoperative $\mathrm{ABG}$ was $5 \mathrm{~dB}$ HL [3]. Some studies of patch materials to treat the TMP are listed in Table 2 [3,15-20].

In the follow-up visit, facial palsy (grade IV according to House-Brackmann facial nerve grading system) occurred in 


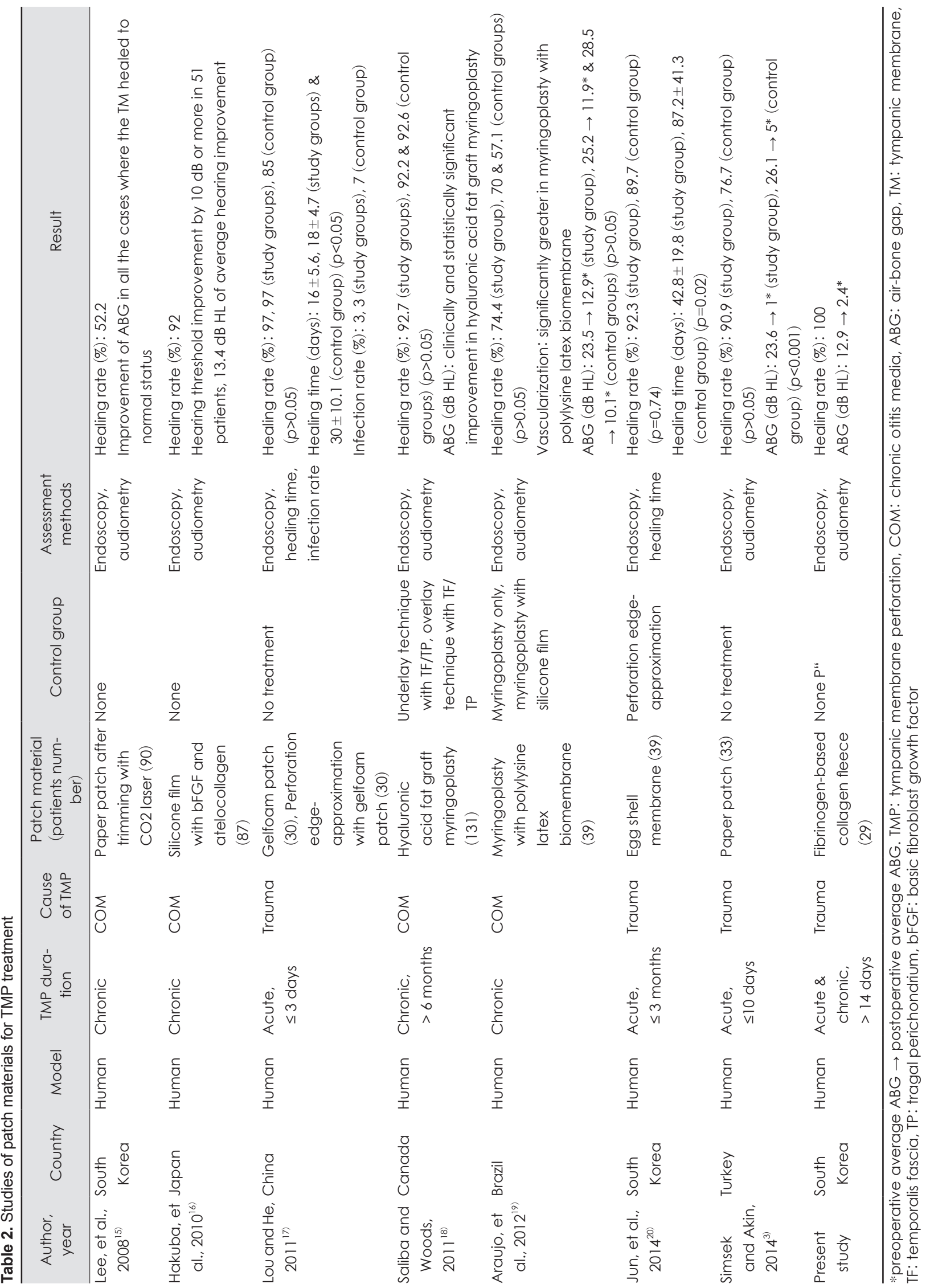


a patient 15 days after FCGM. Methylprednisolone was prescribed for 2 weeks and the patient recovered completely. Two patients had otorrhea after myringoplasty. Bacterial culture of otorrhea showed no growth, and otorrhea disappeared after using oral antibiotics (levofloxacin $250 \mathrm{mg}$ twice a day) in both patients.

The main conclusion of this study is that FCGM may be a viable treatment option in cases of traumatic TMP. However, based on our results, we cannot say that the same procedure may be useful in cases of chronic otitis media. In a recent trial investigating the treatment of chronic and dry TMP, hyaluronic acid fat graft myringoplasty (HAFGM) was an effective technique for TMP treatment. The success rate of HAFGM in this trial was comparable to that of both the underlay and the overlay techniques [18]. However, it was necessary to locally anesthetize via injection and to make an incision in order to harvest fat. In the present study, patients whose perforation had persisted for more the 60 days, or had recurred in spite of the paper patch procedure, showed remarkable improvement. In addition, EAC incisions or injections were not necessary for the procedure, which is simpler and less painful than the HAFGM. We are currently attempting to treat patients with chronic otitis media with dry TMP using FCGM.

In this study, we found that FCGM may be an effective treatment option in traumatic TMP. The postoperative ABG differed significantly from the preoperative. Furthermore, the technique requires no hospitalization, and can be used to avoid traditional tympanoplasty.

\section{Acknowledgments}

This work was supported by a research grant from Jeju National University Hospital.

\section{Conflicts of interest}

The authors have no financial conflicts of interest.

\section{REFERENCES}

1) Villar-Fernandez MA, Lopez-Escamez JA. Outlook for tissue engineering of the tympanic membrane. Audiol Res 2015;5:117.

2) Lindeman P, Edström S, Granström G, Jacobsson S, von Sydow C, Westin T, et al. Acute traumatic tympanic membrane perforations. Cover or observe? Arch Otolaryngol Head Neck Surg 1987;113:1285-7.

3) Simsek G, Akin I. Early paper patching versus observation in pa- tients with traumatic eardrum perforations: comparisons of anatomical and functional outcomes. J Craniofac Surg 2014;25:2030-2.

4) Kim J, Kim CH, Park CH, Seo JN, Kweon H, Kang SW, et al. Comparison of methods for the repair of acute tympanic membrane perforations: Silk patch vs. paper patch. Wound Repair Regen 2010;18: 132-8.

5) Rickenbacher A, Breitenstein S, Lesurtel M, Frilling A. Efficacy of TachoSil a fibrin-based haemostat in different fields of surgery--a systematic review. Expert Opin Biol Ther 2009;9:897-907.

6) Knutsson J, Bagger-Sjöbäck D, von Unge M. Collagen type distribution in the healthy human tympanic membrane. Otol Neurotol 2009; 30:1225-9.

7) Stenfeldt K, Johansson C, Hellström S. The collagen structure of the tympanic membrane: collagen types I, II, and III in the healthy tympanic membrane, during healing of a perforation, and during infection. Arch Otolaryngol Head Neck Surg 2006;132:293-8.

8) Lim DJ. Structure and function of the tympanic membrane: a review. Acta Otorhinolaryngol Belg 1995;49:101-15.

9) Shambaugh GE. Surgery of the ear. 2nd ed. Philadelphia: WB Sunders;1967. p.249-65.

10) Glasscock ME, Gulya AJ. Glasscock-Shambaugh Surgery of the Ear. 5th ed. Hamilton: BC Decker Inc.;2003. p.400-20.

11) Getman V, Devyatko E, Wolner E, Aharinejad S, Mueller MR. Fleece bound sealing prevents pleural adhesions. Interact Cardiovasc Thorac Surg 2006;5:243-6.

12) Hempel JM, Becker A, Müller J, Krause E, Berghaus A, Braun T. Traumatic tympanic membrane perforations: clinical and audiometric findings in 198 patients. Otol Neurotol 2012;33:1357-62.

13) Kristensen S. Spontaneous healing of traumatic tympanic membrane perforations in man: a century of experience. J Laryngol Otol 1992;106:1037-50.

14) Chun SH, Lee DW, Shin JK. A clinical study of traumatic tympanic membrane perforation. Korean J Otolaryngol-Head Neck Surg 1999; 42:437-41.

15) Lee SH, Jin SM, Lee KC, Kim MG. Paper-patch myringoplasty with $\mathrm{CO} 2$ laser for chronic TM perforation. Eur Arch Otorhinolaryngol 2008;265:1161-4.

16) Hakuba N, Iwanaga M, Tanaka S, Hiratsuka Y, Kumabe Y, Konishi $\mathrm{M}$, et al. Basic fibroblast growth factor combined with atelocollagen for closing chronic tympanic membrane perforations in 87 patients. Otol Neurotol 2010;31:118-21.

17) Lou ZC, He JG. A randomised controlled trial comparing spontaneous healing, gelfoam patching and edge-approximation plus gelfoam patching in traumatic tympanic membrane perforation with inverted or everted edges. Clin Otolaryngol 2011;36:221-6.

18) Saliba I, Woods O. Hyaluronic acid fat graft myringoplasty: a minimally invasive technique. Laryngoscope 2011;121:375-80.

19) Araujo MM, Massuda ET, Hyppolito MA. Anatomical and functional evaluation of tympanoplasty using a transitory natural latex biomembrane implant from the rubber tree Hevea brasiliensis. Acta Cir Bras 2012;27:566-71.

20) Jun HJ, Oh KH, Yoo J, Han WG, Chang J, Jung HH, et al. A new patch material for tympanic membrane perforation by trauma: the membrane of a hen egg shell. Acta Otolaryngol 2014;134:250-4. 